\title{
若尔盖沼泽环境中植物和沉积单体正构烷烃 氢同位素组成特征及其成因关系
}

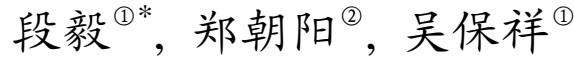 \\ (1) 中国科学院地质与地球物理研究所油气资源研究中心, 兰州 730000 ; \\ (2) 西北大学地质学系, 西安 710069 \\ *E-mail: duany@ns.lzb.ac.cn
}

收稿日期: 2009-06-07; 接受日期: 2010-03-18

国家自然科学基金(批准号: 40772069, 40642007)和国家重点基础研究发展计划(编号: 2005CB422105)资助

\begin{abstract}
摘要为了认识沼泽典型沉积环境中沉积物和现代生物中正构烷烃氢同位素的内在联系, 应用 GC-IRMS 氢同位素分析技术, 对若尔盖沼泽现代草本植物、木本植物叶和沉积物中单体 正构烷烃氢同位素进行了测定. 结果表明, 不同类型植物之间和同一类型植物不同种之间的 正构烷烃氢同位素组成存在着明显的差别, 草本植物正构烷烃的 $\delta \mathrm{D}$ 值 $(-254 \%$ - 184\%o) 要比 木本植物叶类的 $(-195 \%$ - 142\%) 低; 西藏蒿草正构烷烃的氢同位素组成要比早熟禾的轻. 若 尔盖沼泽沉积物中正构烷烃 $\delta \mathrm{D}$ 平均值都反映了它们的生物源为草本植物, 这与研究沼泽泥 炭样品均由草本残骸组成相一致. 不同沉积物样品之间的正构烷烃氢同位素差别, 是由沉积 环境和不同种类草本植物输入引起. 研究发现, 每个样品中单体正构烷烃的 $\delta \mathrm{D}$ 与 $\delta^{13} \mathrm{C}$ 值之 间存在一定的负相关, 并且利用正构烷烃 $\delta \mathrm{D}$ 与 $\delta^{13} \mathrm{C}$ 值相关图可以区分植物种类.
\end{abstract}

关键词

沼泽环境

植物

沉积物 单体正构烷烃

氢同位素

成因关系
单体脂类氢同位素组成可以提供沉积正构烷烃 的成因信息, 因此它在地球化学研究领域中具有广 泛的应用前景 ${ }^{[1 ~ 7]}$. 例如, 认识地质样品中有机质来 源 $^{[5,7 \sim 9]}$ 及研究地区古环境 ${ }^{[1,4,10 ~ 13]}$. 可是, 这种应用研 究特别依赖于不同沉积环境中沉积单体脂类氢同位素 与其生物先质成因关系研究资料的积累. 目前, 虽然 已经对植物中长链正构烷烃的氢同位素进行了一些研 究 ${ }^{[8,12,14 ~ 16]}$, 但是可以利用的现代沼泽植物和沉积有 机质中单体脂类 $\delta \mathrm{D}$ 资料都是有限的, 以致影响了沉 积物单体脂类氢同位素组成的应用研究 ${ }^{[17,18]}$. 另外, 将研究区生物和沉积物中脂类氢同位素组成进行对比 研究, 是建立单体沉积脂类氢同位素与生物先质成因
关系的重要途径, 但是沼泽沉积环境中这种对比研究 还未见报道. 若尔盖沼泽位于青藏高原东部, 气候寒 冷潮湿, 植物主要为草本植物, 木本植物分布极少, 具有特殊的生态环境. 本文研究了若尔盖沼泽草本植 物、木本植物叶和沉积物中单体正构烷烃氢同位素组 成特征, 讨论了沼泽环境沉积单体正构烷烃氢同位素 与其生物先质的成因关系，为沉积单体正构烷烃氢同 位素的应用研究提供依据.

\section{1 样品与分析}

若尔盖沼泽地区在海拔 $3450 \mathrm{~m}$ 左右, 自然生长

引用格式: Duan Y, Zheng C Y, Wu B X. Hydrogen isotopic characteristics and their genetic relationships for individual $n$-alkanes in plants and sediments from Zoigê marsh sedimentary environment. Sci China Earth Sci, 2010, doi: 10.1007/s11430-010-4009-9 
的树木极少, 主要为草本植物, 西藏蒿草是目前若尔 盖沼泽地区的优势植物. 2001 年春天在若尔盖沼泽地 区采集了西藏蒿草、早熟禾和柳树叶样品. 沉积物样 品采自若尔盖沼泽地区花湖附近(样品号为 REG-H-1 至 REG-H-5)和十道班附近(样品号为 REG-S-4), 为现 代沼泽剖面样品, 包括沼泽泥和泥炭, 深度为 0.7 2.0 $\mathrm{m}$. 采样地区海拔 $3450 \mathrm{~m}$, 气候寒冷潮湿, 年均气温 $0.7^{\circ} \mathrm{C}$, 年均降雨量 $650 \mathrm{~mm}$, 相对湿度 $78 \%$. 现在生 长的植物主要为草本植物, 也是形成泥炭的主要物 质.

生物样品首先进行了挑选, 去除杂物, 并用自来 水冲洗, 后用蒸馏水冲洗, 然后其与沉积物都在室温 下自然凉干并粉碎至 100 目, 用二氯甲烷-甲醇 $(2: 1)$ 溶剂进行索氏抽提. 抽提物过滤后蒸发至干, 然后用 氧化铝/硅胶柱色谱进行分离, 饱和烃馏分用正已烷 冲洗, 酮馏分用正己烷 $+10 \%$ 乙酸乙酯冲洗, 醇馏分 用正已烷 $+20 \%$ 乙酸乙酯冲洗, 包含酸性组分的剩余 部分用甲醇溶液冲洗.

饱和烃馏分用美国安捷伦科技公司(Agilent)制 造的色谱-质谱联用仪(GC-MS)进行分析鉴定. MS 为 $5973 \mathrm{~N}$, 离子源温度为 $250^{\circ} \mathrm{C}$, 电离电压为 $70 \mathrm{eV} . \mathrm{GC}$ 为 $6890 \mathrm{~N}$, 色谱柱为 $\mathrm{HP}-5(30 \mathrm{~m} \times 0.32 \mathrm{~mm})$, 固定相涂 膜厚度 $0.25 \mu \mathrm{m}$, 载气为 $\mathrm{He}$, 起始温度 $80^{\circ} \mathrm{C}$, 以 $4^{\circ} \mathrm{C} / \mathrm{min}$ 升温至 $300^{\circ} \mathrm{C}$, 然后恒温 $30 \mathrm{~min}$.

饱和烃馏分在气相色谱-高温热转变-同位素比 值质谱议(GC-TC-IRMS)上进行了单体正构烷烃氢同 位素测定. 气相色谱仪为 Finnigan COMBUSTION III 型, 同位素比值质谱仪为 Delta plus XP 型. 单个化合 物经气相色谱分离后依次进入热解装置, 并在 $1440^{\circ} \mathrm{C}$ 下定量分解转换为 $\mathrm{H}_{2}$. 然后 $\mathrm{H}_{2}$ 进入同位素比值质谱 仪进行氢同位素组成的测定. 色谱柱为 SE-54 (60 $\mathrm{m} \times 0.32 \mathrm{~mm}$ i.d. $\times 0.25 \mu \mathrm{m}$ ) 型熔融石英毛细柱, 载气为 $\mathrm{He}$, 起始温度为 $80^{\circ} \mathrm{C}$, 以 $3^{\circ} \mathrm{C} / \mathrm{min}$ 升到 $300^{\circ} \mathrm{C}$, 然后 恒温 $30 \mathrm{~min}$. 质谱通过调谐使 $\mathrm{H}_{3}$ 因子小于 5 ; 大约每 6 个样品测定一次 $\mathrm{H}_{3}$ 因子, 以观察仪器的稳定性和 保证数据的可靠性. 实验中大约 4 次样品分析测定 1 次已知同位素组成的氢同位素标准(使用经典离线燃 烧法测定 $\mathrm{C}_{14}, \mathrm{C}_{16}, \mathrm{C}_{18}, \mathrm{C}_{23}, \mathrm{C}_{28}$ 和 $\mathrm{C}_{32}$ 共 6 个烷烃化合 物), 以保证氢同位素数据的重现性和准确性. 在每 次样品分析中, 通过引入已知同位素组成的参考氢气 来计算未知样品中单个化合物的氢同位素组成. 每个
样品测定 1 3 次, 所有的氢同位素数据相对于 VSMOW 表达, 在测定化合物具有良好的色谱分离和 适合的强度下, 大多数实测正构烷烃标准偏差 $<5 \%$ o.

\section{2 结果与讨论}

\section{1 植物单体正构烷烃氢同位素组成}

研究样品中单体正构烷烃氢同位素测试结果列 于表 1. 从表 1 中可以看出, 西藏蒿草(RP-1)中正构 烷烃氢同位素组成分布在-251\% - 184\% 之间，平均 为-203\% ; 早熟禾样品(RP-6)中正构烷烃氢同位素组 成分布为 $-254 \%$ - $223 \%$ 之间, 平均为 $-234 \%$ 。 这说 明, 在草本植物样品中, 早熟禾的正构烷烃氢同位素 组成比西藏蒿草的轻. 木本植物叶(RP-4)样品中正构 烷烃氢同位素组成较重, 为-195\% - 142\%。, 平均为 $-171 \%$; 木本植物叶类与 2 个草本植物之间的正构烷 烃平均 $\delta \mathrm{D}$ 值分别相差 32\%o和 63\%。. 它们氢同位素组 成的差异特征与它们碳同位素的相一致, 例如上述 木本植物叶类单体正构烷烃碳同位素组成平均比 2 个草本植物的分别重 $3 \% 0$ 和 $4 \% 0^{[18]}$. 一般来说, 植物 中正构烷烃氢同位素组成受环境因素的影响, 这首 先表现在受控于进入土壤系统的降水同位素, 并且 它随着空间和季节不同而变化; 其次是土壤水和叶 水的蒸发引起同位素的变化, 并且这种变化受气温、 湿度和风速的影响. 所研究的植物都生长在相同的 环境中, 不同种类植物中正构烷烃氢同位素组成明 显的差异可能与植物叶的结构、生长形式和生物化学 分馏有关. 草属于单叶植物, 并且草叶具有长的叶脉; 而树叶具有分支叶脉. 这种叶脉结构差异, 对叶水和 植物组织氢同位素产生重要的影响 ${ }^{[12]}$. 生物体内类 脂物主要由乙酰辅酶 $\mathrm{A}$ 合成, 生物过程中用于还原 的 NADPH(氧化还原辅酶尼克酰胺腺嘌呤二核苷酸) 等还原物质的氢同位素对类脂化合物氢同位素具有 很大的影响, 而不同类型植物在类脂物合成过程中 可能利用了在氢同位素方面具有差别的 $\mathrm{NADPH}^{[19]}$. 另外, 在草和树植物中, 由于树最高, 树叶中水的蒸 发强于草, 而地表(或土壤)水的补给弱于草. 强的蒸 发作用会使树叶中水富集重同位素，而这种水被生 物合成所利用 ${ }^{[20]}$, 这样树叶中正构烷烃氢同位素会 比草的重. 在相同植物中, 不同碳数正构烷烃之间的 $\delta \mathrm{D}$ 值相差 31\% 67\%; 偶碳数正构烷烃的氢同位素 
表 1 生物和沉积物中单体正构烷烃参数及氢同位素组成 $(\% o)^{\mathrm{a})}$

\begin{tabular}{|c|c|c|c|c|c|c|c|c|}
\hline 样品号 & RP-1 & RP-6 & RP-4 & REG-H-1 & REG-H-2 & REG-H-3 & REG-H-5 & REG-S-4 \\
\hline 深度(cm) & - & - & - & $70 \sim 90$ & $130 \sim 150$ & $160 \sim 170$ & $180 \sim 200$ & $180 \sim 200$ \\
\hline 样品类型 & 西藏蒿草 & 早熟禾 & 柳树叶 & 沼泽泥 & 泥炭 & 泥炭 & 泥炭 & 沼泽泥 \\
\hline $\mathrm{C}_{17}$ & & & & -247 & -194 & & -241 & -206 \\
\hline $\mathrm{C}_{18}$ & & & & -180 & -173 & & -237 & -191 \\
\hline $\mathrm{C}_{19}$ & -222 & & & -177 & -200 & & & -204 \\
\hline $\mathrm{C}_{20}$ & & & & -173 & -193 & & & -199 \\
\hline $\mathrm{C}_{21}$ & -251 & & & -195 & -216 & & & -232 \\
\hline $\mathrm{C}_{22}$ & & & -174 & -207 & -215 & -242 & -254 & -206 \\
\hline $\mathrm{C}_{23}$ & -191 & -254 & -184 & -221 & -212 & -225 & -249 & -236 \\
\hline $\mathrm{C}_{24}$ & & & -170 & -213 & -213 & -222 & -248 & -209 \\
\hline $\mathrm{C}_{25}$ & -191 & -250 & -195 & -219 & -213 & -230 & -239 & -222 \\
\hline $\mathrm{C}_{26}$ & & & -149 & -207 & -210 & -210 & -238 & -197 \\
\hline $\mathrm{C}_{27}$ & -184 & -232 & -186 & -207 & -198 & -222 & -237 & -206 \\
\hline $\mathrm{C}_{28}$ & & & & -195 & -190 & -194 & -226 & -197 \\
\hline $\mathrm{C}_{29}$ & -193 & -225 & -142 & -190 & -189 & -215 & -233 & -199 \\
\hline $\mathrm{C}_{30}$ & & & & -180 & -182 & -177 & -223 & -188 \\
\hline $\mathrm{C}_{31}$ & -188 & -223 & & -194 & -198 & -211 & -237 & -201 \\
\hline $\mathrm{C}_{32}$ & & & & -182 & -217 & & -241 & \\
\hline $\mathrm{C}_{33}$ & & -223 & & -194 & -190 & -196 & & -205 \\
\hline 范围 & $-251 \sim-184$ & $-254 \sim-223$ & $-195 \sim-142$ & $-247 \sim-180$ & $-217 \sim-173$ & $-242 \sim-177$ & $-254 \sim-226$ & $-236 \sim-188$ \\
\hline 平均值 & -203 & -234 & -171 & -199 & -200 & -213 & -239 & -206 \\
\hline 烷烃差值 & 67 & 31 & 53 & 67 & 44 & 66 & 28 & 48 \\
\hline $\begin{array}{l}\geqslant \mathrm{C}_{21} \text { 奇碳数 } \\
\text { 平均值 }\end{array}$ & -199 & -234 & -176 & -203 & -203 & -216 & -239 & -214 \\
\hline 平均偶/奇 & 无/-203 & 无/-234 & $-164 /-177$ & $-192 /-205$ & $-199 /-201$ & $-209 /-216$ & $-238 /-239$ & $-198 /-212$ \\
\hline 奇偶平均值差 & & & 12 & 13 & 2 & 7 & 1 & 14 \\
\hline $\mathrm{Paq}$ & & & & 0.19 & 0.31 & 0.63 & 0.53 & 0.59 \\
\hline Pwax & & & & 0.85 & 0.79 & 0.48 & 0.57 & 0.51 \\
\hline
\end{tabular}

a) $\mathrm{Paq}=\left(\mathrm{C}_{23}+\mathrm{C}_{25}\right) /\left(\mathrm{C}_{23}+\mathrm{C}_{25}+\mathrm{C}_{29}+\mathrm{C}_{31}\right) ; \mathrm{Pwax}=\left(\mathrm{C}_{27}+\mathrm{C}_{29}+\mathrm{C}_{31}\right) /\left(\mathrm{C}_{23}+\mathrm{C}_{25}+\mathrm{C}_{27}+\mathrm{C}_{29}+\mathrm{C}_{31}\right)$

略重于奇碳数正构烷烃(表 1). 在 RP-1 样中, $\mathrm{C}_{23}, \mathrm{C}_{25}$, $\mathrm{C}_{27}$ 和 $\mathrm{C}_{29}$ 烷烃几乎有同样的同位素值, 而 $\mathrm{C}_{19}$ 和 $\mathrm{C}_{21}$ 烷烃也具有相似同位素值, 但是这两组烷烃却具有 不同的同位素值. 同一植物中不同碳数正构烷烃的 氢同位素这些变化, 这可能指示它们具有不同的生 化合成机制 ${ }^{[19]}$, 这是因为有机化合物在植物体内的 合成位置及其所使用的酶都对其氢同位素有影响 ${ }^{[2]}$. 同时, 在植物生长过程中, 不同生长条件(如季节差 别)下形成的不同碳数正构烷烃比例的差异, 可以引 起正构烷烃之间的氢同位素变化. 若尔盖沼泽地区 不同季节气温差别较大 $\left(-29.6 \sim 23.6^{\circ} \mathrm{C}\right)$, 会引起植物 不同碳数正构烷烃氢同位素组成的较大差异.

\section{2 沉积单体正构烷烃氢同位素组成}

如表 1 所示, 若尔盖沼泽地区花湖沼泽剖面中, 上层沼泽泥的正构烷烃氢同位素组成分布在 $-247 \%$ o - $180 \%$ 之之间, 平均值为 $-199 \%$; 其下的 3 个
泥炭样品中正构烷烃氢同位素组成分别为 $-217 \%$ o - 173\%o，-242\%o - 177\%。和 -254\%o - $226 \%$,

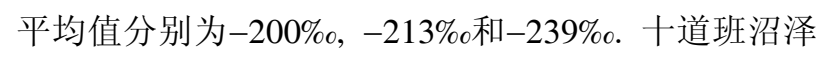
剖面中沼泽泥的正构烷烃氢同位素组成分布在 $-236 \%$ - 188\%。之间, 平均值为 $-206 \%$, 在花湖沼泽 剖面中沉积正构烷烃氢同位素组成范围内, 反映了 若尔盖沼泽不同地区沉积正构烷烃氢同位素组成具 有相似性. 在沼泽沉积样品中, 不同碳数正构烷烃之 间的 $\delta \mathrm{D}$ 值相差 $28 \% 0 \sim 67 \%$; 偶碳数正构烷烃的氢同 位素略重于奇碳数正构烷烃, 两类正构烷烃 $\delta \mathrm{D}$ 值的 平均相差为 $1 \% 00$ 13\%o(表 1), 这些氢同位素特征与所 研究的植物中的氢同位素特征是一致的. 生物化学 研究认为, 沉积物中短链正构烷烃来自菌藻类, 长链 正构烷烃来自高等植物. 可是, 所研究样品中正构烷 烃的氢同位素组成特征表明, 除了上述中等链长的 正构烷氢同位素组成显示了有少量的细菌有机质加 入外, 其他正构烷烃的氢同位素组成未表现出一定 
的规律性, 说明它们具有相似的氢源和生物合成途 径, 因此它们具有类似的成因, 主要来自高等植物, 这与沼泽沉积物中正构烷烃碳同位素研究结果是一 致的 ${ }^{[18]}$.

随着埋深度增加，花湖沼泽剖面中正构烷烃氢 同位素平均值有规律的变轻. 造成这种现象的原因 可能是: (1) 同位素交换反应. 在 0 2.0 m 早期成岩作 用阶段, 若尔盖沼泽成岩水属于淡水, 它的氢同位素 组成要比经过蒸发作用后的草和木本植物叶中的水 轻，通过氢同位素交换反应，可以导致沉积物中正构 烷烃氢同位素组成变轻. 但是, 有机质中氢是以 $\mathrm{C}-\mathrm{H}$ 结合的, 键能较高, 在早期成岩作用阶段的低温条件 下，水难以与碳氢键结合的有机氢发生同位素交换. 因此, 这种可能性比较小. (2) 有机质源不同. 若尔 盖沼泽沉积有机质主要来自草本植物, 由上述可知, 不同草本植物种的氢同位素组成差别明显, 因此不 同地质时期各种草本植物对沉积有机质贡献不同, 可以引起其氢同位素组成差别. (3) 细菌有机质的加 入. 一般来说, 在早期成岩阶段, 随着埋深, 细菌作 用增强; 在若尔盖沼泽早期成岩作用阶段, 可以形成 少量的细菌类并且随着埋深增加, 已被若尔盖沼泽 沉积物的生物标志化合物研究所证实 ${ }^{[21]}$. 这些生物 在生长过程中利用了具有淡水特征的成岩水, 其中 的正构烷烃氢同位素组成相对较轻. 一个明显的证 据是, 在样品中富轻同位素的集中段在 $\mathrm{C}_{22} \sim \mathrm{C}_{25}$ 正构 烷烃之间, 并且在花湖沼泽剖面中, 从浅层样品至深 层样品, $\mathrm{C}_{22} \sim \mathrm{C}_{25}$ 正构烷烃氢同位素组成平均值分别 为 $-215 \%,-213 \%,-230 \%$ 和 $-248 \%$, 它们与其样品 中所有正构烷烃氢同位素平均值相比较, 分别轻 $16 \%$, 13\%o，17\%o和 9\%o, 而这些中等链长的正构烷烃 除了来自高等植物外, 还可以来自细菌 ${ }^{[22]}$. (4) 降水 量的影响. 根据降水量效应, 降水减少, 雨水的 $\delta \mathrm{D}$ 值增加 ${ }^{[23]}$. 因此, 沉积物样品中正构烷烃氢同位素组 成变重也有可能受到降水量的影响.

\section{3 植物与沉积物中单体正构烷烃氢同位素成因 关系}

脂类生物标志化合物氢同位素地球化学研究经 历极短, 但是它在油气地球化学和环境地球化学等 领域已经显示了良好的应用前景. 可是要扩大这种 应用, 必须解决其最基础最关键的科学问题, 即地质 体中脂类生物标志化合物氢同位素的成因特征与机
制. 地质体中单体脂类氢同位素与其母源生物的成 因关系是这一科学问题的关键问题之一，而这一问 题只能通过不同现代沉积环境的生物体和沉积物中 单体脂类生物标志化合物氢同位素的对比, 结合生 物化学分馏和地质历史中环境因素影响的研究去认 识. 沼泽是一个典型的沉积环境, 植物是其有机质的 主要贡献者. 选择该沉积环境中植物和沉积物, 进行 单体正构烷烃氢同位素成因关系的对比研究, 可以 为其应用研究提供科学依据.

为了进行植物和沉积物中正构烷烃氢同位素对 比研究, 计算了长链奇碳数正构烷烃 $\left(\geqslant \mathrm{C}_{21}\right) \delta \mathrm{D}$ 值平 均值, 因为沉积物中长链奇碳数正构烷烃来自高等 植物. 若尔盖沼泽位于中纬度, 现代气候寒冷潮湿, 海拔高, 水体属于淡水, 在这种环境下生长的草本植 物单体长链奇碳数正构烷烃 $\left(\geqslant \mathrm{C}_{21}\right) \delta \mathrm{D}$ 值平均为 $-234 \%$ $-199 \%$, 木本植物叶单体长链奇碳数正构烷 烃平均 $\delta \mathrm{D}$ 值为 $-176 \%$, 可见草本植物长链正构烷烃 具有轻的氢同位素组成. 沼泽沉积物中长链奇碳数 正构烷烃 $\delta \mathrm{D}$ 值平均为-239\% - 203\%o，显示了其氢 同位素组成较轻. 虽然地质历史中环境因素影响当 时植物中正构烷烃氢同位素组成，但是将所研究的 沼泽沉积物中长链奇碳数正构烷烃 $\delta \mathrm{D}$ 值与植物的相 比较, 可以认为草本植物是沉积物中正构烷烃的主 要贡献者, 这与研究沼泽泥炭样品均由草本残骸组 成相一致. 不同沉积物样品之间的正构烷烃氢同位 素差别, 可能是环境因素影响和不同种类草本植物 输入量差别的共同作用结果. 一般来说, 可以用表征 沉积物中水生植物正构烷烃参数 $\mathrm{Paq}=\left(\mathrm{C}_{23}+\mathrm{C}_{25}\right) /\left(\mathrm{C}_{23}+\right.$ $\left.\mathrm{C}_{25}+\mathrm{C}_{29}+\mathrm{C}_{31}\right)$ 和陆生植物正构烷烃参数 $\mathrm{Pwax}=\left(\mathrm{C}_{27}+\mathrm{C}_{29}+\right.$ $\left.\mathrm{C}_{31}\right) /\left(\mathrm{C}_{23}+\mathrm{C}_{25}+\mathrm{C}_{27}+\mathrm{C}_{29}+\mathrm{C}_{31}\right)$ 来估计沼泽环境地质历史中 降水量的变化 ${ }^{[24]}$. 高的 Paq 值和低的 Pwax 值反映了 强的降水量, Paq 值大于 0.4 就指示了水生植物在沼泽 环境中是主要的. 从表 1 中可以看出, 沉积物样品 REG-H-1 和 REG-H-2 中正构烷烃平均 $\delta$ D 值分别为 $-199 \%$ 。和 $-200 \%$, 高于草本植物正构烷烃平均 $\delta \mathrm{D}$ 值. 但是, Paq 值低, 分别为 0.19 和 0.31 ; Pwax 值高, 大于 0.79 , 反映了这个时期降水量小，降水富集重的同位 素, 使这两个样品中正构烷烃具有高的 $\delta \mathrm{D}$ 值. 沉积 物样品 REG-H-3, REG-H-5 和 REG-S-4 中正构烷烃平 均 $\delta \mathrm{D}$ 值分别为 $-213 \%$, $-239 \%$ 和 $-206 \%$, 与草本植 物正构烷烃平均的 $\delta \mathrm{D}$ 值类似. 但是, 这 3 个样品的 $\mathrm{Paq}$ 值分别为 $0.63,0.53$ 和 0.59 , 反映了这个时期降水 
量大, 降水贫重的同位素, 使这些样品中正构烷烃具 有相对低的 $\delta \mathrm{D}$ 值. 同时, REG-H-5 样品 Paq 值低于同 一剖面的 REG-H-3 样品, 可是前者的 $\delta \mathrm{D}$ 值小于后者, 可能说明 REG-H-5 样品中不同种类草本植物的贡献 与 REG-H-3 样品略有不同.

为了从样品中正构烷烃氢和碳同位素组成关系 方面, 认识沼泽沉积物中单体正构烷烃氢同位素的 成因, 我们对沉积物样品 REG-H-1, REG-H-2 和 REG-H-3 中正构烷烃碳同位素进行了分析, 并且结 合以前植物样品正构烷烃碳同位素分析结果 ${ }^{[18]}$, 编 制了样品中正构烷烃 $\delta \mathrm{D}$ 值和 $\delta{ }^{13} \mathrm{C}$ 值相关图. 从图 1 可以看出, 每个样品中正构烷烃 $\delta \mathrm{D}$ 值和 $\delta^{13} \mathrm{C}$ 值之间 存在着一定的负相关性. $\mathrm{Bi}$ 等 ${ }^{[24]}$ 报道了在 $\mathrm{C}_{3}$ 植物中 长链正构烷烃平均 $\delta^{13} \mathrm{C}$ 和 $\delta \mathrm{D}$ 值之间存在着正相关 性, 可是 Chikaraishi 和 Naraoka ${ }^{[25]}$ 在研究植物中正构 烷烃碳氢同位素时没有观察到这种关系. 我们这次 发现植物和沉积物中正构烷烃 $\delta \mathrm{D}$ 值和 ${ }^{13} \mathrm{C}$ 值之间 的负相关性原因还不清楚. 所研究植物样品正构烷 烃 $\delta \mathrm{D}$ 值和 $\delta{ }^{13} \mathrm{C}$ 值的结合使用, 可以明显地将草本和 木本植物区分开, 但是却不能证明沉积物中有机物 的来源. 这表明单体正构烷烃氢同位素与碳同位素 结合, 能更好地研究植物类型, 对于沉积有机生物源 的研究还要结合环境影响因素的研究.

\section{3 结论}

若尔盖沼泽位于青藏高原, 具有寒冷和潮湿的 气候特征; 泥炭形成物质主要为草本植物. 对草本植 物和木本植物叶类中单体正构烷烃氢同位素进行了 测定, 其正构烷烃 $\delta \mathrm{D}$ 分别分布在 $-254 \%$ $184 \%$ 和 $-195 \%$ - $142 \%$ 之间, 平均值分别为 $-234 \%$ $-203 \%$ 。

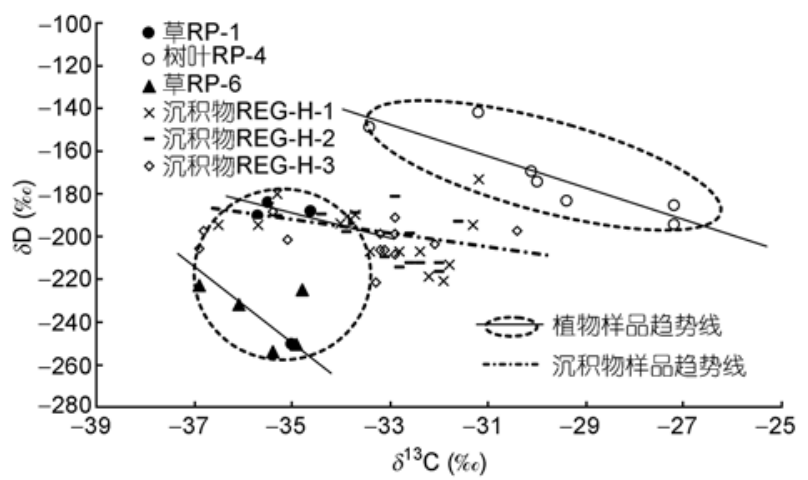

图 1 样品中单体正构烷烃氢和碳同位素组成相关图

和-171\%o，显示了木本植物中正构烷烃富集重同位 素. 植物单体正构烷烃之间的氢同位素变化较大, 差 值分布在 31\%o 67\% 之间; 偶碳数正构烷烃的氢同位 素略重于奇碳数正构烷烃.

沉积单体正构烷烃氢同位素与植物正构烷烃之 间存在一定的成因关系. 沉积物中正构烷烃 $\delta \mathrm{D}$ 值分 布在-254\%o - 173\%。之间，平均值为-239\%o - 199\%o, 主要与所研究的草本植物单体正构烷烃 $\delta \mathrm{D}$ 值更接近, 说明这些样品中正构烷烃主要来自草本植物, 并且 与研究沼泽泥炭均由草本残骸组成相一致. 不同沉 积物样品之间的正构烷烃氢同位素差别, 可能是环 境因素影响和不同种类草本植物输入量差别的共同 作用结果. 研究发现, 每个样品中单体正构烷烃的 $\delta{ }^{13} \mathrm{C}$ 值与 $\delta \mathrm{D}$ 值之间存在一定的负相关性，并且利用 正构烷烃的 $\delta \mathrm{D}$ 与 $\delta^{13} \mathrm{C}$ 值相关图可以区分植物种类. 同时, 沼泽沉积物中正构烷烃的氢同位素组成还与 地质历史中各种环境因素有关，在利用沉积物中正 构烷烃氢同位素组成追溯其母源性质时, 要考虑这 些影响因素。

\section{参考文献}

1 Xie S, Nott C J, Avsejs L A, et al. Palaeoclimate records in compound-specific $\delta$ D values of a lipid biomarker in ombrotrophic peat. Org Geochem, 2000, 31: 1053-1057

2 Sauer P E, Eglnton T I, Hayes J M, et al. Compound-specific D/H ratios of lipid biomarkers from sediments as a peoxy for envieonmental and climatic conditions. Geochim Cosmochim Acta, 2001, 65: 213-222

3 Yang H, Huang Y S. Preservation of lipid hydrogen isotope ratios in Miocene lacustrine sediments and plant fossils at Clarkia, northern Idaho, USA. Org Geochem, 2003, 34: 413-423

4 Sachse D, Radke J, Gleixner G. Hydrogen isotope ratios of recent lacustrine sedimentary $n$-alkanes record modern climate variability. Geochim Cosmochim Acta, 2004, 68: 4877-4889

$5 \mathrm{Li} \mathrm{M} \mathrm{W}$, Huang Y S, Obermajer M, et al. Individual alkanes as a new approach to petroleum correlation: Case studies from the Western Canada Sedimentary Basin. Org Geochem, 2001, 32: 1387—1399 
6 Dawson D, Crice K, Wang S X, et al. Stable hydrogen isotopic compositions of hydrocarbons in torbanites (Late Carbonifierous to Late Permian) deposited under various climatic conditions. Org Geochem, 2004, 35: 189-197

7 熊永强, 耿安松, 潘长春, 等. 陆相有机质中单体烃的氢同位素组成特征. 石油勘探与开发, 2004, 31: 60-63

8 Sachse D, Radke J, Gleixner G. $\delta$ D values of individual $n$-alkanes from terrestrial plants along a climatic gradient- - Implications for the sedimentary biomarker record. Org Geochem, 2006, 37: 469-483

9 Chikaraishi Y, Naraoka H. Carbon and hydrogen isotope variation of plant biomarkers in a plant-soil system. Chem Geol, 2006, 231: 190202

10 Huang Y, Shuman B, Wang Y, et al. Hydrogen isotope ratios of palmitic acid in lacustrine sediments record late Quaternary climate variations. Geology, 2002, 30: 1103-1106

11 Liu W, Huang Y. Compound-specific D/H ratios and molecular distributions of higher plant leaf waxes as novel paleoenvironmental indicators in the Chinese Loess Plateau. Org Geochem, 2005, 36: 851-860

12 Smith F A, Freeman K H. Influence of physiology and climate on $\delta \mathrm{D}$ of leaf wax $n$-alkanes from $\mathrm{C}_{3}$ and $\mathrm{C}_{4}$ grasses. Geochim Cosmochim Acta, 2006, 70: 1172-1187

13 段毅, 吴保祥. 中国大陆主要植物中单体正构烷烃氢同位素组成及其与环境关系研究. 科学通报, 2008, 53: 2776一 2781

14 Chikaraishi Y, Naraoka H, Poulson S R. Hydrogen and carbon isotopic fractionations of lipid biosyntheses among terrestrial $\left(\mathrm{C}_{3}\right.$, $\mathrm{C}_{4}$ and CAM) and aquatic plants. Phytochemistry, 2004, 65: 1369-1381

15 Sessions A L. Seasonal changes in D/H fractionation accompanying lipid biosynthesis in Spatina alterniflora. Geochim Cosmochim Acta, 2006, 70: 2153-2162

16 Pedentchouk N, Sumner W, Tipple B, et al. $\delta^{13} \mathrm{C}$ and $\delta \mathrm{D}$ compositions of $n$-alkanes from modern angiosperms and conifers: An experimental set up in central Washington State, USA. Org Geochem, 2008, 39: 1066-1071

17 段毅, 宋金明, 张辉. 南沙海区生物单体脂类碳同位素研究. 中国科学 D 辑: 地球科学, 2003, 33: 889-894

18 段毅, 张辉, 郑朝阳, 等. 沼泽沉积环境中植物和沉积脂类单体碳同位素组成特征及其成因关系研究. 中国科学 D 辑: 地球科学, 2004, 34: $1151-1156$

19 Sessions A L, Burgoyne T W, Schimmelmann A, et al. Fractionation of hydrogen isotopes in lipid biosynthesis. Org Geochem, 1999, 30: $1193-1200$

20 Craig H, Gordon I, Results Y. Isotopic exchange effects in the evaporation of water, I. Low temperature experimental results. J Geophys Res, 1963, 68: 5079-5087

21 Duan Y, Ma L H. Lipid geochemistry in a sediment core from Ruoergai Marsh deposit (Eastern Qinghai-Tibet plateau, China). Org Geochem, 2001, 32: $1429-1442$

22 Collister J W, Lichtfouse E, Hieshima G, et al. Partial resolution of sources of $n$-alkanes in the saline portion of the Parachute Creek Member River Formation (Piceance Creek Basin, Colorado). Org Geochem, 1994, 21: 645-659

23 卫克勤, 林瑞芬. 论季风气候对我国雨水同位素组成的影响. 地球化学, 1994, 23: 33-41

24 Bi X, Sheng G, Liu X, et al. Molecular and carbon and hydrogen isotopic composition of $n$-alkanes in plant leaf waxes. Org Geochem, 2005, 36: $1405-1417$

25 Chikaraishi Y, Naraoka H. $\delta^{13} \mathrm{C}$ and $\delta$ D relationships among three $n$-alkyl compound classes ( $n$-alkanoic acid, $n$-alkane and $n$-alkanol) of terrestrial higher plants. Org Geochem, 2007, 38: 198-215 